\title{
Appendix 2: List of MOCHA Country Agents
}

http://www.childhealthservicemodels.eu/partnerlisting/country-agents/

\begin{tabular}{ll}
\hline Austria & Dr Reli Mechtler, Lilly Damm \\
Belgium & Dr Sophie Alexander, Dr Liesebeth Borgermans \\
Bulgaria & Prof Vladimir Pilossoff, Dr Georgi Christov \\
Croatia & Dr Ivan Pristas, Dr Marko Brkic \\
Cyprus & Dr Adamos Hadjipanayis \\
Czech Republic & Dr Ales Bourek \\
Denmark & Prof Carsten Obel, Niklas Munksgaard Berg \\
Estonia & Dr Toomas Veidebaum, Eha Nurk, Lagle Suurorg \\
Finland & Prof Mika Gissler \\
France & Dr Christine Edan \\
Germany & Prof Ulrike Ravens-Sieberer, Ann-Katrin Meyrose \\
Greece & Dr Dina Zota \\
Hungary & Dr Péter Altorjai \\
Iceland & Dr Geir Gunnlaugsson \\
Ireland & Carol Hilliard \\
Italy & Dr Roberto Buzzetti \\
Latvia & Prof Anita Villerusa, Irisa Zile \\
Lithuania & Dr Indre Būtienė \\
Luxembourg & Dr Yolande Wagener \\
Malta & Dr Natasha Azzopardi-Muscat \\
Netherlands & A. Prof Danielle Jansen \\
Norway & Prof Anne Karin Lindhal, Dr Ingrid Sperre Saunes \\
Poland & Dr Kinga Zdunek \\
Portugal & Prof Margarida Gaspar de Matos \\
Romania & Dr Maria Roth \\
&
\end{tabular}


(Continued)

Slovakia

Slovenia

Spain

Sweden

United Kingdom
Prof Jozef Suvada

Dr Polonca Truden, Dr Jernej Zavrsnik

Prof Luis Martin Alvarez

Dr Anders Hjern

Prof Mitch Blair, Marcia Philbin, Lisa Cummins 